\title{
Avaliação de espermatozoides caprinos congelados em meio à base de água de coco em pó (ACP-101 ${ }^{\circledR}$ ) ou TRIS
}

\author{
[Evaluation of goat spermatozoa frozen in media based on powder coconut water \\ media based $\left(A C P-101^{\circledR}\right)$ or TRIS]
R.V. Oliveira ${ }^{1}$, J.F. Nunes ${ }^{4}$, C.C.M. Salgueiro ${ }^{4}$, J.M.M. Cavalcante $^{2}$, O.O. Brasil ${ }^{3}$, A.A.A.N. Moura \\ ${ }^{1}$ Aluno de pós-graduação - PDIZ - Universidade Federal do Ceará \\ ${ }^{2}$ Aluno de pós-graduação - PPGCV - Universidade Estadual do Ceará \\ ${ }^{3}$ Aluno de pós-graduação - PPGCA - Universidade de Brasília \\ ${ }^{4}$ Faculdade de Veterinária - Universidade Estadual do Ceará \\ ${ }^{5}$ Departamento de Zootecnia - Universidade Federal do Ceará \\ Campus do Pici - Bloco 810 \\ 60021-970, Fortaleza - CE
}

\begin{abstract}
RESUMO
Compararam-se as características cinéticas e morfológicas de espermatozoides caprinos congelados nos meios à base de ACP- $101^{\circledR}$ e TRIS. Os diluentes utilizados foram: ACP- $101^{\circledR}(+2,5 \%$ gema ovo $+7 \%$ glicerol $) \mathrm{e}$ TRIS (+ 20\% gema ovo $+6,8 \%$ glicerol). Quarenta e oito ejaculados de quatro bodes foram coletados, avaliados, divididos em duas alíquotas e diluídos nos meios ACP-101 ${ }^{\circledR}$ e TRIS, respectivamente, posteriormente congelados e, após 30 dias, descongelados. A avaliação da motilidade espermática por computador foi realizada aos 5, 60 e 120 minutos pós-descongelação. As características de motilidade espermática analisadas foram: motilidade total (MT) $(\%)$ e progressiva (MP) $(\%)$, velocidades média do trajeto do espermatozoide (VAP) $(\mu \mathrm{m} / \mathrm{s})$ e linear (VSL) $(\mu \mathrm{m} / \mathrm{s})$ e população de espermatozoides rápidos (ER) $(\%)$. As avaliações de morfologia espermática quantificaram a porcentagem de espermatozoides normais $(\mathrm{N})$ e as alterações da cabeça (AC), da peça intermediária (API) e do flagelo (AF), aos cinco e 120 minutos pósdescongelação. O diluente TRIS apresentou resultados cinéticos mais elevados que o ACP-101 ${ }^{\circledR}$ aos 60 e 120 minutos pós-descongelação. As AC aos 120 minutos pós-descongelação foram mais altas nos espermatozoides congelados em ACP-101 ${ }^{\circledR}$. Conclui-se que o diluente TRIS promoveu maior viabilidade in vitro dos espermatozoides caprinos pós-descongelação.
\end{abstract}

Palavras-chave: bode, sêmen, criopreservação, diluente

\begin{abstract}
The aim of the work was to compare kinetic and morphologic characteristics of goat sperm frozen in diluent media based on ACP-101 ${ }^{\circledR}$ and TRIS. The employed diluents were: ACP-101 ${ }^{\circledR}(+2.5 \%$ egg yolk $+7 \%$ glycerol) and TRIS (+ $20 \%$ egg yolk $+6.8 \%$ glycerol). Forty eight ejaculates from four bucks were collected, assessed and divided into two aliquots and diluted into the experimental treatments ACP-101 ${ }^{\circledR}$ and TRIS. The samples were frozen and after 30 days, thawed. The computer assisted spermatic motility evaluations were placed into 5, 60 and 120 minutes post-thawing. The motion parameters assessed were: total motility (MT) $(\%)$, progressive motility $(M P)(\%)$, average path velocity $(V A P)(\mu \mathrm{m} / \mathrm{s})$, straight linear velocity $(V S L)(\mu \mathrm{m} / \mathrm{s})$, and population of rapid spermatozoa (ER) (\%). The morphologic parameters: normal spermatozoa $(N)$, head alteration $(A C)$, intermediary piece alteration (API), tail alteration $(A F)$ were evaluated at 5 and 120 min postthawing. The media based on TRIS showed kinetic results significantly superior to $A C P-101^{\circledR}$ at 60 and 120min. After 120min post-thawing the AC was higher in frozen sperm in media based on ACP-101 ${ }^{\circledR}$. The TRIS media promoted better goat spermatozoa in vitro viability post-thawing.
\end{abstract}

Keywords: buck, semen, cryopreservation, extender

Recebido em 28 de julho de 2011

Aceito em 13 de setembro de 2011

E-mail: oliveirarv@ rocketmail.com 


\section{INTRODUÇÃO}

A criopreservação do sêmen é uma biotécnica que deve ser capaz de manter uma população espermática com integridade funcional $\mathrm{e}$ proporcionar adequadas taxas de fertilidade em programas de inseminação artificial. $\mathrm{O}$ processo de congelação promove alterações prejudiciais à funcionalidade dos espermatozoides (sptz) (Salamon e Maxwell, 1995), como danos ultraestruturais, bioquímicos e funcionais, resultando na diminuição da motilidade, viabilidade e fertilidade espermática (Lebouf et al., 2000). Assim, após uma eficiente criopreservação, as células espermáticas devem apresentar motilidade adequada a fim de alcançar o local da fecundação e manter a integridade da membrana espermática para a penetração do oócito (Royere, 1996). Ressalta-se que a composição do diluente é um dos fatores que afeta a proporção de espermatozoides vivos após a congelação e descongelação (Watson, 1995).

Um diluente proposto para a criopreservação seminal deve suprir as células espermáticas com uma fonte de energia, proteção contra criodanos e a manutenção de um ambiente adequado à sobrevivência espermática (Purdy, 2006). Os diluentes à base de leite desnatado (Corteel, 1974) e tris-gema (Salamon e Ritar, 1982; Singh et al., 1995) são os mais utilizados na congelação do sêmen de bodes. Porém, diluentes à base de água de coco in natura têm apresentado resultados satisfatórios quando utilizados para a conservação de espermatozoides de espécies como cães (Cardoso et al., 2003), ovinos (Machado et al., 2006) e caprinos (Salgueiro et al., 2002).

Devido a variações físico-químicas da água de coco e disponibilidade de frutos em estágios ideais, elaborou-se um meio de conservação à base de água de coco em pó $\left(\mathrm{ACP}^{\circledR}\right.$ ACP Biotecnologia, Fortaleza-Ceará, Brasil), caracterizado pela padronização e estabilização da água de coco por meio de um processo de desidratação e subsequente formulação de meios de conservação específicos para células e tecidos (Salgueiro et al., 2002). Meios diluentes à base de $\mathrm{ACP}^{\circledR}$ têm demonstrado viabilidade na criopreservação seminal de cães (Cardoso et al., 2007), suínos (Toniolli et al., 2010) ovinos (Machado et al., 2006), caprinos (Salgueiro et al., 2003) e peixes (Viveiros et al., 2010).
Contudo, a eficiência destes para congelação do sêmen caprino, em específico, ainda precisa ser avaliada com mais precisão em comparação com diluidores atualmente em uso em centrais de inseminação artificial. Diante deste contexto, o presente trabalho teve como objetivo comparar os parâmetros cinéticos e a morfologia de espermatozoides caprinos congelados nos meios à base de água de coco em pó $\left(\mathrm{ACP}^{\circledR}-101\right)$ e TRIS (hidroxi-metil amino metano).

\section{MATERIAL E MÉTODOS}

Foram utilizados quatro bodes de três a cinco anos de idade e mais de $70 \mathrm{~kg}$ de peso vivo, dois da raça Saanen e dois da raça Bôer. Os animais foram mantidos em confinamento, em baias individuais, alimentados com feno de tifton (Cynodon sp.), 500g de ração concentrada, com $18 \%$ de proteína bruta, e sal mineral e água à vontade.

$\mathrm{O}$ diluente à base de água de coco em pó (ACP101) ${ }^{\circledR}$, com pH 6,8 e $300 \mathrm{mOsm} / \mathrm{kg}$, foi constituído de água de coco em pó acrescida de tampões, $100 \mathrm{~mL}$ de água destilada, 40mg de gentamicina, $2,5 \%(\mathrm{v} / \mathrm{v})$ de gema de ovo e $7 \%$ (v/v) de glicerol. O diluente foi dividido em duas frações: "fração A" sem glicerol e "fração B" com $14 \%(\mathrm{v} / \mathrm{v})$ de glicerol (Salgueiro et al., 2003).

O diluente à base de TRIS, com $\mathrm{pH}$ 6,8 e $300 \mathrm{mOsm} / \mathrm{kg}$, foi constituído por: $3,786 \mathrm{~g}$ de Tris-hidroximetil-aminometano, 2,11g de ácido cítrico monohidratado, $100 \mathrm{~mL}$ de água destilada, $40 \mathrm{mg}$ de gentamicina, $1 \%$ de frutose, $20 \%$ (v/v) de gema de ovo e $6,8 \%(\mathrm{v} / \mathrm{v})$ de glicerol. O diluente foi dividido em duas frações: " $A$ " sem glicerol e "B" com 13,6\% (v/v) de glicerol (Singh et al., 1995).

O sêmen foi coletado por meio de vagina artificial $\left(\mathrm{IMV}^{\circledR}\right.$ Technologies, França), na presença de uma fêmea em cio, induzido por uma injeção de $1 \mathrm{mg}$ (IM) de benzoato de estradiol (Estrogin ${ }^{\circledR}$, Farmavet, Brasil) 48 horas antes do procedimento, sendo realizadas duas coletas semanais, obtendo 12 amostras por animal, o que totalizou 48 ejaculados. Após cada coleta, os ejaculados foram mantidos em banhomaria a $30^{\circ} \mathrm{C}$ enquanto se realizou a avaliação seminal. 
O julgamento de motilidade massal (MM) foi realizado observando-se sob microscopia óptica convencional uma gota de sêmen puro em lâmina, atribuindo notas de 0 a 5 . Quanto às análises de porcentagem de espermatozoides móveis (\%) (PEM) e à motilidade individual progressiva (0-5) (MIP), estas ocorreram pela avaliação de uma gota de sêmen diluído sob lâmina coberta com lamínula por microscopia óptica convencional, e foram qualificadas em porcentagem e valores de 1 a 5 , respectivamente (Chemineau et al., 1991). Para análise da morfologia espermática, foram confeccionados esfregaços de sêmen corados com eosinanigrosina para avaliação de 200 células quanto à porcentagem de espermatozoides normais $(\mathrm{N})$, alterações da cabeça (AC), peça intermediária (API) e flagelo (AF) sob microscopia óptica de contraste de fase (Colas, 1980).

Após a avaliação da MM, PEM, MIP e morfologia espermática, cada ejaculado foi dividido em duas alíquotas iguais, sendo, então, acondicionadas em tubos de ensaio de $15 \mathrm{~mL}$. Estas formaram dois tratamentos experimentais: ACP-101 ${ }^{\circledR}$ e TRIS, sendo submetidas a uma prédiluição na taxa de 1:2 (sêmen:fração A diluente) $(\mathrm{v} / \mathrm{v})$, a $30^{\circ} \mathrm{C}$. A concentração espermática foi mensurada pelo método de espectrofotometria, (Chemineau et al., 1991).

Após a avaliação da concentração espermática e os cálculos para diluição, as alíquotas foram novamente diluídas a $30^{\circ} \mathrm{C}$ por meio da adição do volume restante da fração " $A$ " de cada diluidor. Posteriormente, os tubos de ensaio contendo as amostras seminais diluídas foram acondicionados em um recipiente plástico contendo $200 \mathrm{~mL}$ de água a $30^{\circ} \mathrm{C}$. Este, em seguida, foi refrigerado durante 120 minutos até alcançar $4^{\circ} \mathrm{C}$, a uma taxa de $-0,22^{\circ} \mathrm{C} /$ minuto. Uma vez a $4^{\circ} \mathrm{C}$, realizou-se a terceira diluição adicionando-se o volume da fração "B", de cada diluente em três etapas, com intervalos de cinco minutos, até uma concentração final de $400 \times 10^{6} \mathrm{sptz} / \mathrm{mL}$, com mais cinco minutos de tempo de equilíbrio.

O sêmen diluído e resfriado a $4^{\circ} \mathrm{C}$, de todos os grupos, foi envasado em palhetas de $0,25 \mathrm{~mL}$ $\left(\right.$ IMV $^{\circledR}$ Technologies, França) (com 100×10 espermatozoides cada). As palhetas foram, então, colocadas em rampa de congelação, distante $3,5 \mathrm{~cm}$ do nível do nitrogênio líquido, onde se congelaram nos vapores $\left(-100^{\circ} \mathrm{C}\right)$ por 10 minutos, sendo posteriormente submersas em nitrogênio líquido $\left(-196^{\circ} \mathrm{C}\right)$ e em seguida armazenadas em botijão criogênico.

Trinta dias após a congelação, as palhetas foram descongeladas em banho-maria a $37^{\circ} \mathrm{C}$, durante 30 segundos. Posteriormente foram acondicionadas em tubos Eppendorf ${ }^{\circledR}$ (Standard Micro Test Tube 3810, Eppendorf ${ }^{\circledR}$, Alemanha), e mantidas a $37^{\circ} \mathrm{C}$ por 120 minutos para realização do teste de termorresistência (Chemineau et al., 1991; CBRA, 1998). As avaliações de motilidade espermática foram realizadas aos cinco, 60 e 120 minutos pósdescongelação. Para rediluição pósdescongelação, utilizaram-se os respectivos diluentes baseados em ACP- $101^{\circledR}$ ou TRIS sem gema e sem glicerol. Três palhetas de cada tratamento por ejaculado foram descongeladas, sendo cada uma avaliada e rediluída até uma concentração de $20-50 \times 10^{6} \mathrm{sptz} / \mathrm{mL}$. Assim, $5 \mu \mathrm{L}$ da amostra rediluída era colocada em câmara de Makler $^{\circledR}$ (Sel-Medical Instruments, Israel), sob aquecimento a $37^{\circ} \mathrm{C}$, para as análises de motilidade espermática.

A cinética espermática foi analisada com o auxílio de microscópio de contraste de fases acoplado a uma vídeo-câmara adaptada ao sistema Sperm Class Analyser ${ }^{\circledR}$ (SCA, Microptic S.L., versão 3.2.0, Espanha), mensurando-se as seguintes variáveis: motilidade total (MT;\%), motilidade progressiva (MP;\%), velocidade média do trajeto do espermatozoide (VAP; $\mu \mathrm{m} / \mathrm{s}$ ), velocidade linear (VSL; $\mu \mathrm{m} / \mathrm{s}$ ) e a população de espermatozoides rápidos (ER;\%). A morfologia espermática pós-descongelação foi avaliada aos cinco e 120 minutos pósdescongelação, como descrito previamente.

Para a análise estatística, foi utilizado o delineamento experimental de blocos ao acaso em parcelas subdivididas, sendo cada bloco representado por um bode, os diluidores considerados as parcelas e os tempos de incubação pós-descongelação as subparcelas. As variáveis significativas na análise de variância, pelo procedimento GLM do programa estatístico SAS (SAS Institute Inc, Cary, NC, EUA), foram submetidas ao teste Duncan $(\mathrm{P}<0,05)$ para comparação de médias. 


\section{RESULTADOS E DISCUSSÃO}

Os dados relacionados com a avaliação da motilidade auxiliada por computador de espermatozoides caprinos diluídos e congelados em meios à base de água de coco em pó (ACP$101^{\circledR}$ ) e TRIS após cinco minutos do teste de termorresistência estão ilustrados na Tab. 1. Não houve efeito do diluente em relação às motilidade total (MT) e progressiva (MP). Entretanto, o diluente TRIS apresentou melhores resultados em relação aos espermatozoides rápidos (ER), velocidades linear (VSL) e média da trajetória (VAP). No presente trabalho, não foi observado efeito do diluente sobre os parâmetros de motilidade total (MT), progressiva (MP) e espermatozoides morfologicamente normais (N) aos cinco minutos pósdescongelação, mesmo o diluente ACP-101 apresentando oito vezes menos gema de ovo na sua composição que o meio diluente TRIS. Ressalta-se que estes dados de motilidade total e espermatozoides normais constatados aos cinco minutos foram semelhantes aos obtidos por Dorado et al. (2007) utilizando dois dos principais diluentes para congelação sêmen caprino, leite desnatado e TRIS, com $20 \%$ gema. Os resultados de motilidade progressiva (MP) espermática aos cinco minutos pósdescongelação, quantificados no presente estudo, foram semelhantes aos obtidos por outros autores congelando sêmen caprino em diluentes à base de TRIS com gema de ovo (Hidalgo et al., 2006; Amidi et al., 2010). Houve efeito do diluente sobre os valores de VAP, VCL e ER independentemente do período de incubação pósdescongelação, tendo o diluente à base de TRIS apresentado resultados superiores ao ACP-101 para estas avaliações. A qualidade da motilidade espermática, que se expressa pelos parâmetros relacionados à velocidade dos espermatozoides, foi preservada de forma mais eficiente no diluente TRIS. Contudo, os resultados de VAP e VCL obtidos no tratamento ACP-101, aos cinco minutos pós-descongelação, foram mais altos que os obtidos por Amidi et al. (2010), que utilizaram o diluidor TRIS-gema-glicerol para congelação de sêmen caprino.

Avaliações cinéticas dos espermatozoides foram processadas após 60 minutos do teste de termorresistência conforme dados da Tab. 2. O diluente à base de TRIS apresentou resultados cinéticos significativamente superiores ao ACP$101^{\circledR}$. Na Tab. 3, são apresentados os resultados das avaliações cinéticas processadas após 120 minutos do teste de termorresistência. $\mathrm{O}$ efeito do diluente se manteve, em que os espermatozoides diluídos-congelados em TRIS apresentaram valores superiores em relação aos parâmetros cinéticos. Os dados obtidos com a avaliação morfológica de espermatozoides caprinos diluídos e congelados em meios à base de água de coco em pó (ACP-101 ${ }^{\circledR}$ ) e TRIS, após cinco minutos do teste de termorresistência, podem ser observados na Tab. 4. Não houve diferença significativa na porcentagem de espermatozoides normais e alterações morfológicas entre os diluentes à base de ACP$101^{\circledR}$ e TRIS aos cinco minutos pósdescongelação. As principais alterações morfológicas após descongelação do sêmen constatadas foram relacionadas às alterações de cabeça e de flagelo. Ressalta-se que a maioria das alterações de cabeça observadas após a descongelação do sêmen estava relacionada às alterações acrossomais. Vários trabalhos têm constatado o efeito deletério da congelação sobre a porcentagem de anormalidades acrossomais em espermatozoides caprinos (Singh et al., 1995; Ferrari e Barnabe, 1999; Azeredo et al., 2001; Hidalgo et al., 2006).

Tabela 1. Análise computadorizada da motilidade de espermatozoides caprinos diluídos e congelados em meios à base de água de coco (ACP-101 ${ }^{\circledR}$ ) e TRIS, após cinco minutos do teste de termorresistência

\begin{tabular}{lccccc}
\hline Diluidor & $\begin{array}{l}\text { MT } \\
(\%)\end{array}$ & $\begin{array}{l}\text { MP } \\
(\%)\end{array}$ & $\begin{array}{c}\text { ER } \\
(\%)\end{array}$ & $\begin{array}{c}\text { VSL } \\
(\mu \mathrm{m} / \mathrm{s})\end{array}$ & $\begin{array}{c}\text { VAP } \\
(\mu \mathrm{m} / \mathrm{s})\end{array}$ \\
\hline ACP-101 $^{\circledR}$ & $54,5 \pm 16,5$ & $27,1 \pm 11,3$ & $18,7 \pm 11,5 \mathrm{~b}$ & $40,4 \pm 10,4 \mathrm{~b}$ & $47,4 \pm 11,8 \mathrm{~b}$ \\
TRIS & $54,6 \pm 16,3$ & $30,9 \pm 12,5$ & $27,5 \pm 12,5 \mathrm{a}$ & $47,9 \pm 10,5 \mathrm{a}$ & $55,6 \pm 10,6 \mathrm{a}$
\end{tabular}

Letras diferentes na coluna indicam diferenças entre os diluidores $(\mathrm{P}<0,05)$; motilidade total (MT;\%) e progressiva (MP;\%), velocidade média do trajeto do espermatozoide (VAP; $\mu \mathrm{m} / \mathrm{s})$ e linear (VSL; $\mu \mathrm{m} / \mathrm{s})$ e população de espermatozoides rápidos (ER; \%). 
Tabela 2. Análise computadorizada da motilidade de espermatozoides caprinos diluídos e congelados em meios à base de água de coco (ACP-101 ${ }^{\circledR}$ ) e TRIS, após 60 minutos do teste de termorresistência

\begin{tabular}{lccccc} 
Diluidor & $\begin{array}{l}\text { MT } \\
(\%)\end{array}$ & $\begin{array}{c}\text { MP } \\
(\%)\end{array}$ & $\begin{array}{c}\text { ER } \\
(\%)\end{array}$ & $\begin{array}{c}\text { VSL } \\
(\mu \mathrm{m} / \mathrm{s})\end{array}$ & $\begin{array}{c}\text { VAP } \\
(\mu \mathrm{m} / \mathrm{s})\end{array}$ \\
\hline ACP-101 ${ }^{\circledR}$ & $17,0 \pm 15,4 \mathrm{~b}$ & $4,7 \pm 5,6 \mathrm{~b}$ & $2,7 \pm 4,0 \mathrm{~b}$ & $15,0 \pm 11,4 \mathrm{~b}$ & $20,18 \pm 13,89 \mathrm{~b}$ \\
& & & & & \\
TRIS & $43,5 \pm 19,4 \mathrm{a}$ & $13,9 \pm 8,3 \mathrm{a}$ & $21,1 \pm 11,6 \mathrm{a}$ & $32,2 \pm 8,6 \mathrm{a}$ & $45,4 \pm 11,7 \mathrm{a}$
\end{tabular}

Letras diferentes na coluna indicam diferença entre diluidores $(\mathrm{P}<0,05)$; motilidade total $(\mathrm{MT} ; \%)$ e progressiva (MP;\%), velocidade média do trajeto do espermatozoide (VAP; $\mu \mathrm{m} / \mathrm{s}$ ) e linear (VSL; $\mu \mathrm{m} / \mathrm{s}$ ) e população de espermatozoides rápidos (ER; \%).

Tabela 3. Análise computadorizada da motilidade de espermatozoides caprinos diluídos e congelados em meios à base de água de coco (ACP-101 ${ }^{\circledR}$ ) e TRIS, após 120 minutos do teste de termorresistência

\begin{tabular}{lccccc}
\hline Diluidor & $\begin{array}{l}\text { MT } \\
(\%)\end{array}$ & $\begin{array}{c}\text { MP } \\
(\%)\end{array}$ & $\begin{array}{c}\text { ER } \\
(\%)\end{array}$ & $\begin{array}{c}\text { VSL } \\
(\mu \mathrm{m} / \mathrm{s})\end{array}$ & $\begin{array}{c}\text { VAP } \\
(\mu \mathrm{m} / \mathrm{s})\end{array}$ \\
\hline ACP-101 ${ }^{\circledR}$ & $1,1 \pm 3,7 \mathrm{~b}$ & $0,4 \pm 1,7 \mathrm{~b}$ & $0,2 \pm 0,9 \mathrm{~b}$ & $2,5 \pm 9,6 \mathrm{~b}$ & $3,0 \pm 11,1 \mathrm{~b}$ \\
TRIS & $34,1 \pm 18,3 \mathrm{a}$ & $9,7 \pm 6,5 \mathrm{a}$ & $15,4 \pm 10,3 \mathrm{a}$ & $27,0 \pm 10,3 \mathrm{a}$ & $38,3 \pm 14,4 \mathrm{a}$
\end{tabular}

Letras diferentes na coluna indicam diferenças entre os diluidores $(\mathrm{P}<0,05)$; motilidade total (MT;\%) e progressiva (MP;\%), velocidade média do trajeto do espermatozoide (VAP; $\mu \mathrm{m} / \mathrm{s}$ ) e linear (VSL; $\mu \mathrm{m} / \mathrm{s})$ e população de espermatozoides rápidos $(\mathrm{ER} ; \%)$.

Tabela 4. Morfologia de espermatozoides caprinos diluídos e congelados em meios à base de água de coco $\left(\right.$ ACP- $\left.101^{\circledR}\right)$ e TRIS, após cinco minutos do teste de termorresistência

\begin{tabular}{lcccc} 
Diluidor & $\begin{array}{c}\mathrm{N} \\
(\%)\end{array}$ & $\begin{array}{c}\mathrm{AC} \\
(\%)\end{array}$ & $\begin{array}{c}\text { API } \\
(\%)\end{array}$ & $\begin{array}{c}\text { AF } \\
(\%)\end{array}$ \\
\hline ACP-101 ${ }^{\circledR}$ & $64,9 \pm 8,1$ & $27,7 \pm 8,2$ & $1,3 \pm 1,19$ & $6,1 \pm 3,41$ \\
TRIS & $62,4 \pm 9,8$ & $31,3 \pm 9,7$ & $0,9 \pm 1,16$ & $5,4 \pm 3,33$
\end{tabular}

Letras diferentes na coluna indicam diferenças entre os diluidores $(\mathrm{P}<0,05)$; porcentagem de espermatozoides normais $(\mathrm{N} ; \%)$, alterações da cabeça (AC;\%), peça intermediária (API;\%) e flagelo (AF;\%).

Os dados referentes à avaliação morfológica de espermatozoides caprinos diluídos e congelados em meios à base de água de coco em pó (ACP$101^{\circledR}$ ) e TRIS, após 120 minutos do teste de termorresistência estão ilustrados na Tab. 5. Após 120 minutos do teste de termorresistência, a porcentagem de espermatozoides normais foi superior no meio diluente à base de TRIS.

O período de incubação de 120 minutos a $37{ }^{\circ} \mathrm{C}$ após a descongelação também afetou negativamente a morfologia espermática, aumentando significativamente as porcentagens de alterações de cabeça em ambos os diluentes, sendo a porcentagem de alterações de cabeça aos 120 minutos superior no diluente à base de ACP quando comparada ao diluente à base de TRIS. Salgueiro et al. (2003), ao compararem espermatozoides caprinos congeladosdescongelados em meios diluentes à base de água de coco in natura e TRIS-gema-glicerol, não observaram diferenças significativas relacionadas às alterações morfológicas entre os diluentes. 
Oliveira et al.

Tabela 5. Morfologia de espermatozoides caprinos diluídos e congelados em meios à base de água de coco em pó (ACP-101 ${ }^{\circledR}$ ) e TRIS, após 120 minutos do teste de termo-resistência

\begin{tabular}{|c|c|c|c|c|}
\hline Diluidor & $\begin{array}{c}\mathrm{N} \\
(\%)\end{array}$ & $\begin{array}{l}\mathrm{AC} \\
(\%)\end{array}$ & $\begin{array}{l}\text { API } \\
(\%)\end{array}$ & $\begin{array}{l}\text { AF } \\
(\%)\end{array}$ \\
\hline ACP-101 ${ }^{\circledR}$ & $48,9 \pm 7,7 b$ & $44,1 \pm 9,8 \mathrm{a}$ & $0,9 \pm 1,5$ & $6,1 \pm 4,4$ \\
\hline TRIS & $56,7 \pm 6,6 a$ & $37,7 \pm 6,8 b$ & $0,7 \pm 1,0$ & $4,9 \pm 4,1$ \\
\hline
\end{tabular}

Letras diferentes na coluna indicam diferenças entre os diluidores $(\mathrm{P}<0.05)$; porcentagens de espermatozoides normais $(\mathrm{N} ; \%)$, alterações da cabeça (AC;\%), peça intermediária (API;\%) e flagelo (AF;\%).

O processo de criopreservação do sêmen caprino tem um efeito deletério sobre as motilidades total e progressiva e sobre a velocidade média da trajetória (Sundaraman e Edwin, 2006). Tuli e Holtz (1995) calcularam o percentual de redução de motilidade progressiva subjetiva do sêmen caprino fresco para o pós-descongelado em torno de $46 \%$. Estudos relacionados à composição de novos diluentes para criopreservação visam aumentar a viabilidade espermática pósdescongelação. Recentemente, foram realizados estudos, no Brasil, de avaliação de meios à base de água de coco em pó para preservação de células espermáticas. O ACP manteve os mesmos constituintes bioquímicos da água de coco in natura, tendo a vantagem de ser facilmente estocado, transportado e preparado (Viveiros et al., 2010). Dessa forma, meios à base de ACP surgem como opções viáveis para a congelação seminal de bodes.

$\mathrm{O}$ meio à base de TRIS possuiu maior quantidade de gema, diferença que pode ter promovido sua maior proteção às crioinjúrias. Neste estudo, observou-se que os espermatozoides caprinos congelados em meio à base de $\mathrm{ACP}-101^{\circledR}$ contendo $2,5 \%$ de gema apresentaram valores de motilidade, aos 60 e 120 minutos de incubação a $37^{\circ} \mathrm{C}$, mais baixos que o diluente à base de TRIS, o qual possuía $20 \%$ de gema. Recentemente, foi demonstrado que a avaliação da porcentagem de espermatozoides móveis aos 120 min póscongelação possui caráter preditivo para a fertilidade via inseminação artificial cervical em caprinos (Furstoss et al., 2010). Outros trabalhos constataram no sêmen caprino descongelado uma baixa termorresistência quando congelado em diluente à base de TRIS com 2,6\% gema (Ferrari e Barnabé, 1999; Azêredo et al., 2001). A gema de ovo é um crioprotetor externo, adicionado aos diluentes seminais, visando a uma proteção não apenas relacionada aos danos oriundos do resfriamento (Purdy, 2006), como também às crioinjúrias produzidas pela etapa de congelação (Aboagla e Terada, 2004). Trabalhos têm indicado que, dentre os constituintes da gema de ovo, a lipoproteína de baixa densidade apresenta efeito protetor aos criodanos (Watson, 1976). Bergeron et al. (2004) demonstraram que a lipoproteína de baixa densidade (LDL) presente na gema de ovo previne a ligação de proteínas BSPs ao espermatozoide e efluxos de fosfolipídios da membrana espermática, tendo um efeito benéfico para a criopreservação espermática.

\section{CONCLUSÕES}

O diluente à base de TRIS promoveu maior viabilidade in vitro de espermatozoides caprinos quando comparado ao diluente ACP-101 ${ }^{\circledR}$. Os parâmetros cinéticos, após cinco minutos de incubação a $37^{\circ} \mathrm{C}$, de espermatozoides caprinos diluídos e congelados em meio diluente à base de ACP $-101^{\circledR}$ demonstram a potencialidade deste diluidor na congelação seminal de bodes.

\section{AGRADECIMENTOS}

Os autores agradecem ao apoio recebido pela CAPES, CNPq, FUNCAP e FUNDECI.

\section{REFERÊNCIAS BIBLIOGRÁFICAS}

AMIDI, F.; FARSHAD, A.; KHOR, A.K. Effects of cholesterol-loaded cyclodextrin during freezing step of cryopreservation with TCGY extender containing bovine serum albumin on quality of goat spermatozoa. Cryobiology, v.61, p.94-99, 2010.

ABOAGLA, E.M.E.; TERADA, T. Effects of egg yolk during the freezing steps of cryopreservation on the viability of goat spermatozoa. Theriogenology, v.62, p.11601172, 2004. 
AZÊREDO, G.A.; ESPER, C.R.; RESENDE, K.T. Evaluation of plasma membrane integrity of frozen-thawed goat spermatozoa with or without seminal plasma. Small Ruminant Res., v.41, p.257-263, 2001.

BERGERON, A.; CRÊTE, M.H.; BRINDLE, Y. et al. Low-density lipoprotein fraction from hen's egg yolk decreases the binding of the major proteins of bovine seminal plasma to sperm and prevents lipids efflux from the sperm membrane. Biol. Reprod., v.70, p.708-717, 2004.

CARDOSO, R.C.S.; SILVA, A.R.; UCHOA, D.C. et al. Cryopreservation of canine semen using a coconut water extender with egg yolk and three different glycerol concentrations. Theriogenology, v.59, p.743-751, 2003.

CARDOSO, R.C.; SILVA, A.R.; SILVA, L.D. et al. Evaluation of fertilizing potential of frozenthawed dog spermatozoa diluted in ACP-106 using an in vitro sperm oocyte interaction assay. Reprod. Dom. Anim., v.42, p.11-16, 2007.

CBRA. Colégio Brasileiro de Reprodução Animal. Manual para exame andrológico $e$ avaliação de sêmen animal. 2.ed. Belo Horizonte: 1998. 49p.

CHEMINEAU, P.; CAGNIÉ, Y.; GUÉRIN, Y. Training manual on artificial insemination in sheep and goats. Rome: FAO, 1991. 222p.

COLAS, G. Variations sazonnieres de la qualité du sperme chez le bélier Ile-de-France II-étude de la morphologie cellulaire et de la motilité massale. Reprod. Nutr. Dev., v.20, p.1789-1799, 1980.

CORTEEL, J.M. Viabilité des spermatozoids de bouc conserves et congelés avec ou sans leur plasma seminal: effet du glucose (viability of goat spermatozoa deep frozen with or without seminal plasma: glucose effect). Ann. Biol. Anim. Bioche. Biophys., v.14, p.741-745, 1974.

DORADO, J.; RODRÍGUEZ, I.; HIDALGO, M. Cryopreservation of goat spermatozoa: Comparison of two freezing extenders based on post-thaw sperm quality and fertility rates after artificial insemination. Theriogenology, v.68, p.168-177, 2007.

FERRARI, S.; BARNABE, V.H. Effect of two kinds of diluents and two freezing methods on caprine semen quality. Braz. J. Vet. Res. Anim. Sci., v.36, p.1-4, 1999.
FURSTOSS, V.; BORDERES, F.; FORGERIT, Y. et al. The value of the percentage of motile sperm in predicting a significant portion of the fertility variation of frozen-thawed buck semen. Theriogenology, v.74, p.1197-1206, 2010.

HIDALGO, M.; RODRIGUEZ, I.; DORADO, J. Influence of staining and sampling procedures on goat sperm morphometric using the sperm class analyzer. Theriogenology, v.66, p.996-1003, 2006.

LEBOEUF, B.; RESTALL, B.; SALAMON, S. Production and storage of goat semen for artificial insemination. Anim. Reprod. Sci., v.62, p.113-141, 2000.

MACHADO, V.P.; NUNES, J.F.; ARAÚJO, A.A. et al. Fertility following intracervical or intrauterine laparoscopic insemination of ewes using extenders based on coconut water. Braz. J. Vet. Res. Anim. Sci., v.43, p.43-49, 2006.

PURDY, P.H. A review on goat sperm cryopreservation. Small Ruminant Res., v.63, p.215-225, 2006.

ROYERE, D.; BARTHELEMY, C.; HAMAMAH S. Cryopreservation of spermatozoa: a review. Hum. Reprod. Update, v.2, p.553-559, 1996.

SALAMON, S.; RITAR, A.J. Deep freezing of Angora goat semen: effects of diluent composition and method and rate of dilution on survival of spermatozoa. Aust. J. Biol. Sci., v.35, p.295-303, 1982.

SALAMON, S.; MAXWELL, W.M.C. Frozen storage of ram semen II. Causes of low fertility after cervical insemination and methods of improvement. Anim. Reprod. Sci., v.38, p.1-36, 1995.

SALGUEIRO, C.C.M.; NUNES, J.F.; OLIVEIRA, K.P.L. et al. Utilization of extenders based on coconut water "in natura" and in powder on the does artificial insemination at fixed time. Braz. J. Anim. Reprod., v.5, p.96-98, 2002.

SALGUEIRO, C.C.M.; NUNES, J.F.; MATEOS-REX, E. et al. Evaluation quality of goat semen after thawing by Hyposmotic Test. Braz. J. Anim. Reprod., v.27, p.377-378, 2003. 
SINGH, M.P.; SINHA, A.K.; SINGH, B.K. Effect of cyoprotectants on certain seminal attributes and on the fertility of buck spermatozoa. Theriogenology, v.43, p.10471053, 1995.

SUNDAMARAN, M.N.; EDWIN, M.J. Cryopreservation of Boer goat spermatozoa during cryopreservation cycle. Online J. Vet. Res., v.10, p.117-124, 2006.

TONIOLLI, R.; TONIOLLO, G.H.; FRANCESHINI, P.H. et al. Uso do diluente água de coco em pó (ACP-103®) na conservação prolongada do sêmen do varrão: avaliação in vitro e in vivo. Arq. Bras. Med. Vet. Zootec., v.62, p.1072-1079, 2010.

TULI, R.K., HOLTZ, W. Effect of season on the freezability of Boer goat semen in the northern temperate zone. Theriogenology, v.43, p.13591363, 1995.
VIVEIROS, A.T.M. NASCIMENTO, A.F.; ORFÃO, L.H. et al. Motility and fertility of the subtropical freshwater fish streaked prochilod (Prochilodus lineatus) sperm cryopreserved in powdered coconut water. Theriogenology, v.74, p.551-556, 2010.

WATSON, P.F. The protection of ram and bull spermatozoa by the low density lipoprotein fraction of egg yolk during storage at $5^{\circ} \mathrm{C}$ and deep freezing. J. Thermal Biol., v.1, p.137-141, 1976.

WATSON, P.F. Recent developments and concepts in the cryopreservation of spermatozoa and assessment of their post-thawing function. Reprod. Fertil. Dev., v.7, p.871-891, 1995. 